\title{
Batch Repowering Scheme in Rechargeable Wireless Sensor Networks
}

\author{
Wei Shang, Yijun Wang* \\ Logistics Department, \\ China Tobacco Zhejiang Industrial Co., Ltd. \\ Hangzhou China \\ e-mail: steve860910@aliyun.com
}

\author{
Yuliang Li \\ Manufacturing Department, \\ China Tobacco Zhejiang Industrial Co., Ltd. \\ Hangzhou, China \\ e-mail: liyuliang@zjtobacco.com
}

\begin{abstract}
Energy optimization of WSN is very important to prolong the lifetime of tiny and energy insufficient sensor nodes. Traditional LEACH-like energy saving approaches try to balance the load of every node, which prove to be very effective. Since, these approaches suppose that the sensor nodes are disposable; and when the lifetime ends, the network system will be shut down. This is not suitable for the rechargeable sensor node applications any more. To decrease the cost of replacement of rechargeable sensor nodes, a tradeoff of amount of spare sensor nodes and labor should be made deliberately. In this paper, a batch repowering scheme is proposed to solve this type of problem. The scheme is designed to balance the energy insufficient nodes and make them run out of energy at the same time, so that they can be replaced in batch. This idea is just opposite to the LEACH-like algorithms. Simulations represent that our scheme is optimal in enhance system reliability with low cost for reutilized WSN applications.
\end{abstract}

Keywords- WSN; rechargeable sensor network; batch repowering scheme; energy efficiency; robust system; energy hole

\section{INTRODUCTION}

Energy management is one of the most important problems a wireless sensor network has to confront. Think about numerous tiny wireless sensor nodes with compact computation and communication resources are scattered in a wide range of areas, it is a nightmare at all to pick out and replace the energy depletion nodes.

To prolong the lifetime of a wireless sensor network, the most effective method is to balance the energy load of every node. Because of the intrinsic unbalance load of sensor nodes due to different requirement of data acquisition, computation and distance of communication, part of nodes in the network dying prematurely always lead to connectivity breaks among adjacency nodes and thereby form holes, or in other terms, such as blind spots, isolated nodes, and so on, which indicate the loss of information may be critical for certain tasks.

To date, there are hundreds of energy balance researches. As one of the pioneer works, the low-energy adaptive clustering hierarchy protocol, which is now well-known as LEACH protocol [1], sheds light on the solution of clustering. In $\mathrm{LEACH}$, sensor nodes are divided into clusters and a cluster head node is selected randomly to undertake the task of data convergence and communication with sink stations. Since the cluster heads are most energy-intensive nodes, they are selected in turn in different rounds, so that every node in the network is decaying nearly synchronously till the entire network dies out.

LEACH clustering protocol has proved an effective method to prolong lifetime of wireless sensor networks theoretically and in engineering. Many improved methods are proposed afterwards.

LEACH supposes that sensor nodes are homogeneous of energy status. But in most practical applications, the initial energy capacity of every node is not equal. Khalil [2] et al. takes heterogeneous-aware energy consumption into account and introduces an evolutionary model, named as SAERP, to ensure longer stability period and shorter instability period.

The cluster head node of LEACH is elected randomly. This strategy should lead to early death of some unfortunate nodes. Asaduzzaman [3] proposes a modified cross layer cluster head node election approach to obtain a diversity order of $\mathrm{M}$ cluster head in each cluster in long distance communication. Lee's research [4] shows that the selection of the lowest total energy consumption node as data a data aggregation node to send data to a sink is superior to other cluster head schemes.

Sensor nodes in LEACH are stationary. Nasser [5] improves it with a zone-based routing protocol for mobile sensor networks, which is most common in applications like habitat monitoring or search and rescue.

In LEACH, every non-cluster head node selects the cluster according the distance to the cluster head node individually, so that the cluster size is not expected. Li [6] develops a method to optimize the cluster size according to distance between cluster head and sink to solve "energy hole" problem.

There are also lots of two-tiered sensor network topologic improvements of LEACH clustering approach. Bari [7, 8] proposes two fast and efficient integer linear program formulations for assigning sensor nodes to clusters in a two-tiered network, where the relay nodes are used as cluster heads. And then he also proposes a genetic algorithm to quickly gain optimal and energy-aware routing in large networks $[9,10]$.

LEACH supposes that there is only one sink station to aggregate sensor data. It seems that multiple sink stations will improve the performance directly. Kim [11] optimizes the positions of multiple sink stations with a mixed integer linear programming model. Shi $[12,13]$ designs an optimal 
approximation algorithm to reduce an infinite search space to a finite-element search space for sink station location. Marta [14] proposes a sink station moving scheme to prevent energy holes near sinks. Azad [15] jointly optimizes the base station locations by maximize the lifetime bound using genetic algorithm.

Routing protocol is also improvable of LEACH. Mo [16] and Qin [17] proposes the top-k querying algorithm not only in cluster formation phase and also in inter-cluster choosing phase to establish quick routing path.

Traditionally, the wireless sensor networks are applied for military purpose, of which disposable sensor nodes seem acceptable. However, in the past few years, with the rapid development of internet of things, many novel applications of wireless sensor network are promoted, such as environmental monitoring, precision agriculture, sensor logistics, and so on. In these applications, sensor nodes are not disposable but reused while taking account of system cost.

Nowadays, battery technology of self-power is not an issue any more. Solar cells, piezoelectric [18], thermoelectric energy harvester [19, 20], and microware energy transmission technology [21] are used to repower remote sensor nodes. However, these self-power technologies need outside energy source, which may not available at any time and place. Rechargeable battery is still a fine choice of energy recovery of sensor nodes.

With the conventional energy balance methods, all the nodes should die together ultimately. The surge workload of repowering the entire network must be tremendous and the application system must be shut down during the repowering period. An alternative method for the decision maker to accelerate the repowering process is to replace all the energy exhausted nodes with full charged spare ones, which will increase largely the application system cost.

To avoid this, a novel energy configuration scheme should be improved to balance replacement schedule. There has been little attention to this problem yet. In this paper, a batch repowering scheme is proposed to replace energy depleted sensor nodes in batches and in turn to maintain the application network system operating smoothly with relatively small quantitative spare sensor nodes.

The structure of this paper is as follow: Section 2 describes the primary radio energy dissipation model, optimal batch repowering scheme and its assumptions. Section 3 brings out the implementation of batch repowering scheme, including candidate cluster head selection, flowchart and cluster generation. Section 4 represents some numerical simulations.

\section{PRELIMINARY}

\section{A. The Radio Energy Dissipation Model}

Here, we use the classical simple model of radio energy dissipation in reference [22]. To transmit a k-bit message a distance $d$, the radio energy dissipation of the send node is:

$$
E_{T x}(k, d)=E_{\text {elec }} \cdot k+\varepsilon_{\text {amp }} \cdot k \cdot d^{2} .
$$

And to receive this message, the radio energy dissipation of the receive node is:

$$
E_{R x}(k, d)=E_{\text {elec }} \cdot k .
$$

Where $E_{\text {elec }}$ and $\varepsilon_{\text {amp }}$ are constants and we assume $E_{\text {elec }}=50 \mathrm{~nJ} / \mathrm{bit}$ and $\varepsilon_{\text {amp }}=100 \mathrm{pJ} / \mathrm{bit} / \mathrm{m} 2$.

\section{B. Assumptions}

The assumptions in this study are stated as follow:

1 . We suppose that all the sensor nodes have the equal amount of initial energy of E0 and the full charged spare sensor nodes also have the energy of E0.

2. The communication among sensor nodes is divided into rounds as same as LEACH. And each round is divided into two phases: set-up phase and steady-state phase. In the set-up phase, the cluster head nodes are elected, each nonhead node selects cluster according to the distance to the elected head nodes, and routine path of each node is settled down. The steady-state phase contains several periods. In each period, sensor nodes are woken up and finish communication once and then return sleeping mode.

3. There is only one base station for data aggregation.

4. All the sensor nodes are homogeneous not only in the initial energy but also in the energy dissipation direction.

5. The total number of sensor nodes in the network system dose not change and the position of each sensor node and base station does not change too.

6 . Only single hop routing is considered to simplify the problem.

7. The recharging period of sensor nodes is not concerned. In the practical applications, recharging might be time consuming. So batch recharging might need a certain amount of charging equipment for parallel operation.

8. Data of every sensor node need to be transmitted to the sink station in every phase is equal.

\section{The Optimal Batch Repowering Scheme}

First of all, we write $\mathrm{L}$ as the average number of sensor nodes for a unit labor cost to pick back energy exhausted nodes; $\mathrm{C}$ as the cost of a sensor node and $\mathrm{M}$ as the number of spare sensor nodes for replacing energy depleted ones.

So the labor cost of every replacement is $\mathrm{l}=\mathrm{M} / \mathrm{L}$. Because in most times, the labor can not divided into fraction, that is, $\mathrm{l}$ is always an integer, so the optimal value of $\mathrm{M}$ is closer to an integral multiple of $\mathrm{L}$. The cost of spare nodes $c=M \cdot C$, which is an investment only once. It is obvious that more spare nodes lead to more spare nodes cost and less replacement frequency, which will simplify management of labor, but increase the unreliability of the network system. So a suitable spare nodes reservation is the precondition of the optimizing scheme.

Then, with $\mathrm{M}$ spare sensor nodes, we try to realize two aims: one is during every replacement period, there are $\mathbf{M}$ on-system sensor nodes run out of energy and need to be replaced; and the other is replacement of $\mathrm{M}$ sensor nodes will not lead to shut down the entire network system or disconnect other sensor nodes for a long time, which will cause system unreliable. That is the number of depleted nodes will not be larger than $\mathrm{M}$.

So, in one certain replacement period, the less remaining energy sensor nodes are more inclined to be selected as 
heavy burden cluster head nodes. This is just opposite to the traditional energy balance schemes.

We write $\bar{E}_{\text {head }}$ as the total energy consumed by cluster head nodes during a replacement period on average. This criterion is used to select the candidate set of nodes to be replaced at the end of the current replacement period.

At the beginning of one replacement period, the remaining energy of every sensor node is ordered. To select $\mathrm{M}$ head nodes among the lowest remaining energy nodes, we must notice that the nodes of remaining energy less than $\bar{E}_{\text {head }} / M$ will probably die out in advance, if they are selected as cluster head nodes. There is only one way to solve this situation. That is, to replace all the other $\mathrm{M}$ candidate nodes ahead of schedule.

So, for the sake of assurance, we select $M$ least remaining energy nodes satisfied with $E_{\text {remain }} \geq \bar{E}_{\text {head }} / M$ as the candidate cluster nodes of the coming replacement period. It is easy to estimate $\bar{E}_{\text {head }}$ using the accumulation energy consuming of former replacement periods.

The least remaining energy nodes of $E_{\text {remain }}<\bar{E}_{\text {head }} / M$ will be non-head nodes yet. If they run out of energy after the replacement period, they will be replaced too and the relative more energy candidate nodes are left to be replaced in next period..

\section{III.THE IMPLEMENTATION OF BATCH REPOWERING SCHEME}

\section{A. Candidate Cluster Head Nodes Set Generation Approach}

The crucial step of batch repowering scheme is the candidate cluster head nodes selection. As shown in Fig. 1, after several replacement periods, the energy distribution of sensor nodes in the network begin to diverge. Although in a distribution environment, each sensor node can not know others' parameters, they can transfer their own status to the sink station actively in the end of replacement periods. The order of remaining energy calculation and the selection procedure can be done in the sink station.

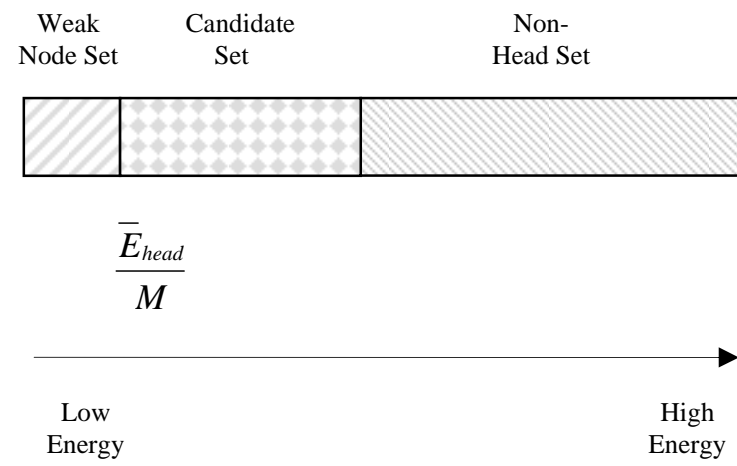

Figure 1. Candidate cluster head nodes selection of batch repowering scheme

Sensor nodes are ordered by remaining energy from low to high. The weak node set whose remaining energy less than $\bar{E}_{\text {head }} / M$ can not be selected as cluster head. And the nodes of candidate set are those remaining energy greater than $\bar{E}_{\text {head }} / M$ low energy nodes.

\section{B. Flowchart of Batch Repowering Scheme}

As shown in Fig. 2, the relationship of replacement period and round is one replacement period contains several rounds. And a round contains only one set-up phase and several steady-phases respectively.

At the beginning of a replacement period, the candidate set is selected according to the rules described in section 3.1. It is obvious that the initial energy of candidates might be closer but uneven and the position of them might be dispersion or convergence. To balance the energy of candidates and minimize the total energy consumption, the cluster size and component non-head nodes are varied in different set-up phase of every round.

It is not assurance that there will not be any accidents, such as node faults or node energy exhausted prematurely and so on, which should lead to "hole" and information or functioning loss. In order to enhance the robust of the entire network system, theses accident nodes should be replaced separately in emergency, which will cause extra labor costs. However, the amount of spare nodes does not increase because $\mathrm{M}$ spare nodes are full recharged after last replacement.

It is noticed that after candidates exhausted, the replaced nodes are $\mathrm{M}$ least energy nodes but not candidate ones. The reason is that there might be non-head nodes exhausted too. The remnant of candidates is left to the next replacement period.

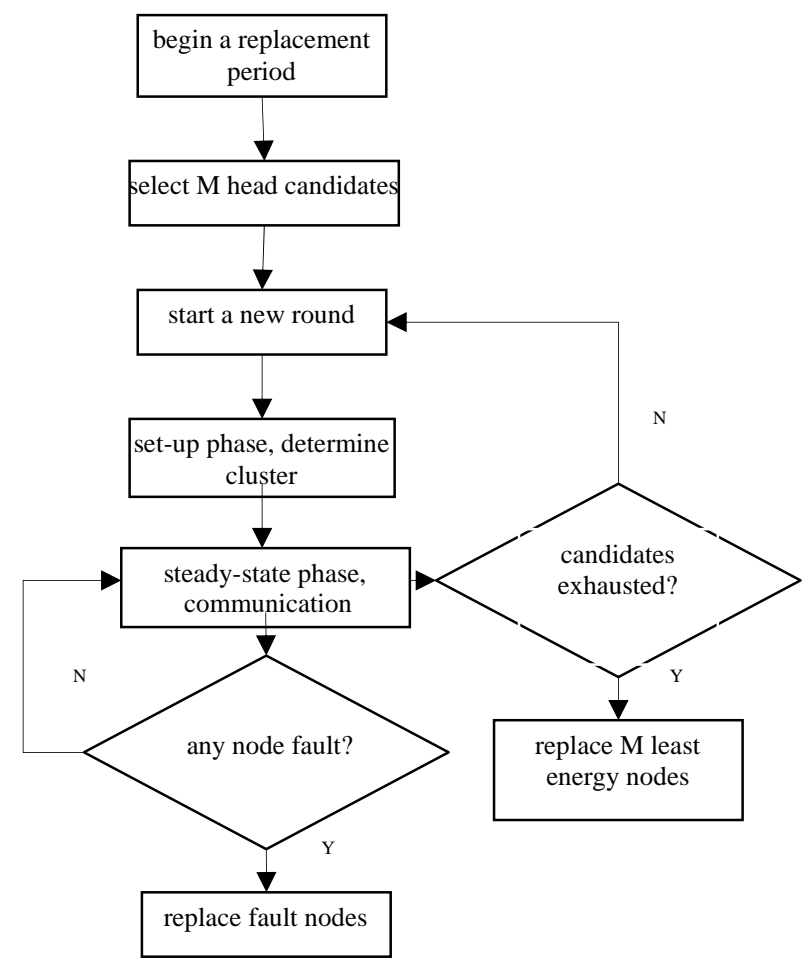

Figure 2. Flowchart of batch repowering scheme 
A replacement period contains several rounds, which contains one set-up phase and several steady-state phase individually. After selecting head candidates, every noncluster node decides which cluster it will join in according to the distance, candidates' energy balance and aggregating direction to the sink station, differently in each set-up phase. After certain communication in steady-state phases, two statuses of candidates exhausted and any node fault will trigger replacement. Replacing fault nodes is the emergency operation which will cause extra labor costs, but is good to the system robust.

\section{Optimize the Selection Cluster of Non-head nodes}

According to equation (1) and (2), to receive message there is an elementary energy related to data size, while to transmit message need extra energy related to the distance. As shown in Fig. 3, there are two different cases of the single hop routine.

Suppose A is a non-head node. If A directly transmit a unit message to sink station $\mathrm{S}$, the transmitting energy is:

$$
E^{A S}=E_{T x}^{A S}=E_{\text {elec }}+\varepsilon_{\text {amp }} \cdot|A S|^{2} .
$$

Here, we don't concern the energy consumption of sink station, because it is always power supplied with wires.

If there are two cluster head node adjacent to A, A wonders which cluster it will join in? Cluster head node B, A and sink station $\mathrm{S}$ make up an acute triangle, while cluster node $\mathrm{C}, \mathrm{A}$ and $\mathrm{S}$ make up an obtuse triangle.

In the acute triangle $\triangle A B S$, the single hop route of node A transmit a unit message to sink station $S$, the total energy consumption of node $A$ and $B$ is:

$$
E^{\triangle A B S}=E_{T x}^{A B}+E_{R x}^{A B}+E_{T x}^{B S}=3 E_{\text {elec }}+\varepsilon_{\text {amp }} \cdot\left(|A B|^{2}+|B S|^{2}\right) \text {. }
$$

In the obtuse triangle $\triangle A C S$, the single hop route of node A transmit a unit message to sink station $\mathrm{S}$, the total energy consumption of node A and B is:

$$
E^{\triangle A C S}=E_{T x}^{A C}+E_{R x}^{A C}+E_{T x}^{C S}=3 E_{\text {elec }}+\varepsilon_{\text {amp }} \cdot\left(|A C|^{2}+|C S|^{2}\right) .
$$

It is obvious that $E^{\triangle A C S}<E^{\triangle A B S}$. So to decrease the total energy consumption of the entire network system, non-head node A prefers to join in head node C's cluster. However, there might be so many component nodes in head node C's cluster, which will lead to the energy shortage of $\mathrm{C}$. So, to balance the load of $\mathrm{A}$ and $\mathrm{B}$, an optimal cluster size determining algorithm will be discussed next.

How about the idea of non-head node A communicating with sink station S directly? Compare equation (3) and (4), we can obtain that $E^{A S}<E^{\triangle A B S}$. That is, in the acute triangle, direct communication cost less total energy. However, the precondition is A can reach S or A is energy rich.

Compare equation (3) and (5), we can obtain that $E^{\triangle A C S}<E^{A S}$, only when the distances are large enough to countervail double times of basic energy consumption.

Finally, node A burdens heavier in direct path; node B burdens heavier in acute triangle $\triangle A B S$; and node $C$ burdens heavier in obtuse triangle $\triangle A C S$, which will be considered in the energy assignment of clusters.

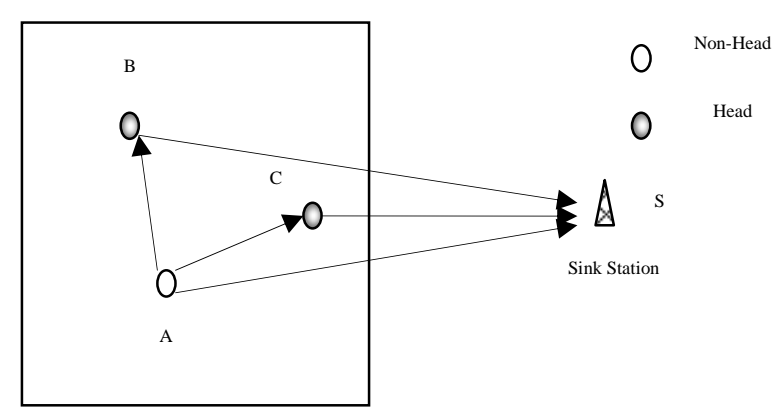

Figure 3. Comparing different cases of single hop routine

There are three paths non-head node A wants to transmit message to sink station S: (1) direct path; (2) single hop route through head node $\mathrm{B}$; (3) single hop route through head node $\mathrm{C}$.

As shown in Fig. 4, from the view of candidate head nodes, the cluster size will influence its energy consumption. So a global assignment mechanism should be introduced to balance the cluster size. Opposite to the LEACH-like balance algorithms which select sufficient energy nodes as cluster head, insufficient energy nodes should probably lead to energy failure without deliberate cluster assignment.

So, the remaining energy of every candidate node is sent to the sink station in the beginning of a replacement period and is ordered there. Then, quota of each candidate node is generated according to the proportion of its remaining energy to the total summation of all the candidate nodes' remaining energy. The quota is sent back to the candidate node afterward.

When an adjacent non-head node wants to join in the cluster, it will check the quota of the cluster head node firstly. If it is used up, the applier should turn to other adjacent clusters.

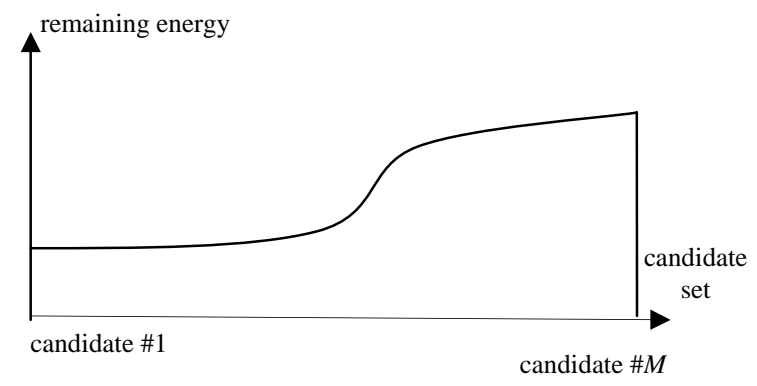

Figure 4. Remaining energy distribution of the candidate set

The remaining energy distribution of the candidate set is used to determine the cluster size of every head node. The remaining energy is sent to the sink station and then ordered, since each candidate node doesn't know the whole situation of network. And quota of each candidate node is sent back afterward. When the non-head node wants to join in the cluster, it will check whether the quota of the head node is used up. 


\section{NUMERICAL SIMULATIONS}

\section{A. Simulation Setup}

For our simulations, we used a 100-node network where nodes were randomly distributed between $(x=0, y=0)$ and $(x=100, y=100)$ with the sink station at location $(x=50$, $y=175$ ), which is as same as LEACH reference [1]. For simplification, each data message is 500 bytes and the energy consumption of the cluster set-up will be omitted. The initial energy of each sensor node and the recharge full energy is equal to be $\mathrm{E} 0=2 \mathrm{~J}$. We suppose that a labor can replace 5 nodes in a unit of work time and the labor cost will be the ceiling of fraction, that is, if the requirement of replacement is less than 5 nodes, the labor still need be paid as 1 unit of work time. So, we set that 1 labor cost (LC) is equal to 5 replacements. Similarly, we set that a spare sensor node cost (SC) is equal to 1 sensor node.

\section{B. Simulation Results and Discussion}

Firstly, we compare our scheme with other 3 routing approaches: (1) LEACH; (2) direct routing; (3) random routing. According to Ref. [1], cluster number between 3 and 5 is most energy efficient; we implement LEACH approach with 5 clusters and set about 20 steady-state phase per round. It is reported in Ref. [1] that after about 20 rounds, death nodes begin to appear and there is no any node alive in the network just over 30 rounds.

We modify LEACH approach to a rechargeable one with a little change, that is, as soon as the node is exhausted, a full recharged node should be replaced in the same place of the exhausted one. The same replacement strategy is applied in the above-mentioned (2) and (3) situation.

In direct routing case, we suppose all the nodes communicate to the sink station directly and individually. We plan to use this case as a benchmark.

In random routing case, we suppose all the nodes either probably communicate to the sink station directly, or probably single hop by another node in a random manner. We plan to use this case to simulate the characteristics of the network all in free and uncontrolled status.

And at last, we set the amount of spare sensor node of our scheme is $M=5$. And the node will be replaced only if its remaining energy $e \leq 0$ in the other three cases. The initial value of threshold $\bar{E}_{\text {head }} / M$ is 0 . We set the steady-state phase as a minim time interval for the sake of simplicity. The replacement period set-up time and cluster set-up phase time are not immerged into timeline. We do 10000 times simulation and suppose that the sensor network monitor 100 times per day.

As shown in Fig. 5, replacement of LEACH is concentrated in several days and the amount is large. Replacement of direct route is almost less than 10, which indicate that direct route is efficient if the sink station can be reached directly. Replacement of random route is about 5 and increases at the end of 100 days. In batch repowering scheme, only 5 and 10 replacements, so maximum of 10 spare sensor nodes need to be stored.
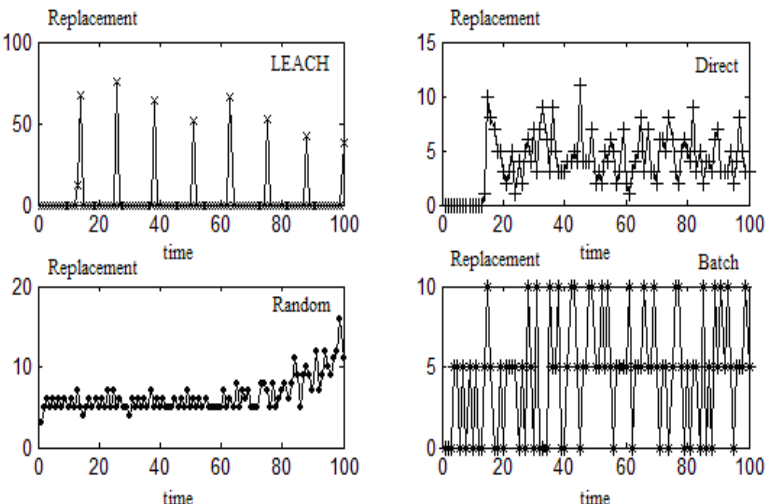

Figure 5. The amount of replacement nodes in every day of four cases

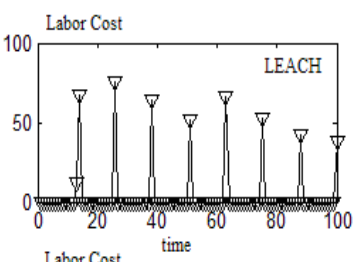

Labor Cost
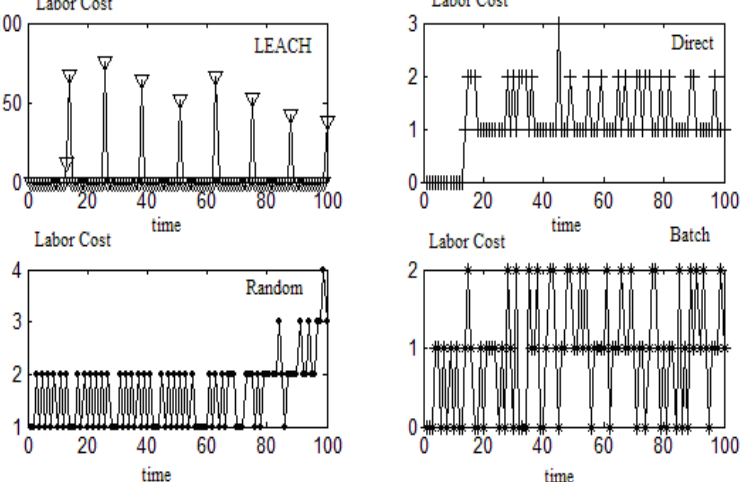

Figure 6. Labor cost of replacement in every day of four cases

As shown in Fig. 6, labor cost of four cases is different. LEACH needs a great deal of labor but only in several certain days. Direct routine and random routine needs 1 or 2 labors regularly. And batch repowering scheme needs 1 or 2 labors too.

And the total replacement amounts of four cases are: LEACH(470); direct routine(394); random routine(641); batch repowering scheme(455). The total labor cost of four cases are: LEACH(470); direct routine(112); random routine(163); batch repowering scheme(91).

So, our batch repowering scheme is optimal in replacement amounts and labor cost than other approaches. And this scheme can be used in a real application of wireless grain depot surveillance system..

\section{CONCLUSIONS}

There are hundreds of energy balance algorithms of wireless sensor network over the past decade. However, they all suppose that the sensor nodes can be thrown away after its energy runs out. Although the sensor node is tiny and cheap, nowadays, according to the system cost and robust, rechargeable sensor nodes should be re-concerned with. Batch repowering scheme proposed in this paper is designed for this purpose which is little concerned yet. We hope our primary work will attract similar research focus in the near future. 


\section{ACKNOWLEDGMENT}

This work is partly supported by the project of Science and Technology Department of Zhejiang Province, China (Grant No.2013C11066), project of China Tobacco Zhejiang Industrial Co., Ltd.(Grant No. ZJZY2013E001) and Scientific Research Fund of Zhejiang Provincial Education Department (Grant No.Y201223754).

\section{REFERENCES}

[1] W. B. Heinzelman, A. P. Chandrakasan and H. Balakrishnan. An Application-Specific Protocol Architecture for Wireless Microsensor Networks. IEEE Transactions on Wireless Communications. 2002, 1(4): 660-670.

[2] Khalil, E. A. and B. A. Attea. Stable-Aware Evolutionary Routing Protocol for Wireless Sensor Networks. Wireless Personal Communications. 2013, 69(4): 1799-1817.

[3] Asaduzzaman and H. Y. Kong. Energy Efficient Cooperative LEACH Protocol for Wireless Sensor Networks. Journal of Communications and Networks. 2010, 12(4): 358-365.

[4] Lee, E., S. Park, et al. On Selection of Energy-Efficient Data Aggregation Node in Wireless Sensor Networks. IEICE Transactions on Communications. 2010, E93B(9): 2436-2439.

[5] Nasser, N., A. Al-Yatama, et al. Zone-Based Routing Protocol with Mobility Consideration for Wireless Sensor Networks. Telecommunication Systems. 2013, 52(4): 2541-2560.

[6] Li, H., Y. L. Liu, et al. COCA: Constructing Optimal Clustering Architecture to Maximize Sensor Network Lifetime. Computer Communications. 2013, 36(3): 256-268.

[7] Bari, A., A. Jaekel, et al. Clustering Strategies for Improving the Lifetime of Two-Tiered Sensor Networks. Computer Communications. 2008, 31(14): 3451-3459.

[8] Bari, A., A. Jaekel, et al. Optimal Placement and Routing Strategies for Resilient Two-Tiered Sensor Networks. Wireless Communications \& Mobile Computing. 2009, 9(7): 920-937.

[9] Bari, A., S. Wazed, et al. A Genetic Algorithm Based Approach for Energy Efficient Routing in Two-Tiered Sensor Networks. Ad Hoc Networks. 2009, 7(4): 665-676.

[10] Bari, A., A. Jaekel, et al. Energy-Efficient Design Techniques for
Large-scale, Two-tiered Sensor Networks. Ad Hoc \& Sensor Wireless Networks. 2011, 11(1-2): 137-164.

[11] Kim, H., T. Kwon, et al. Multiple Sink Positioning and Routing to Maximize the Lifetime of Sensor Networks. IEICE Transactions on Communications. 2008, E91B(11): 3499-3506.

[12] Shi, Y., Y. T. Hou, et al. Algorithm Design for a Class of Base Station Location Problems in Sensor Networks. Wireless Networks. 2009, 15(1): 21-38.

[13] Shi, Y. and Y. T. Hou. Optimal Base Station Placement in Wireless Sensor Networks. ACM Transactions on Sensor Networks. 2009, 5(4).

[14] Marta, M. and M. Cardei. Improved Sensor Network Lifetime with Multiple Mobile Sinks. Pervasive and Mobile Computing. 2009, 5(5): 542-555.

[15] Azad, A. P. and A. Chockalingam. Enhancing Lifetime of Wireless Sensor Networks Using Multiple Data Sinks. International Journal of Sensor Networks. 2011, 9(3-4): 139-157.

[16] Mo, S. F., H. Chen, et al. Clustering-Based Routing for Top-k Querying in Wireless Sensor Networks." EURASIP Journal on Wireless Communications and Networking. 2011.

[17] Qin, Z. Q., C. Ma, et al. An Overlapping Clustering Approach for Routing in Wireless Sensor Networks. International Journal of Distributed Sensor Networks. 2013.

[18] Yoon Y. J., Park W. T., and Li K. H. H., et al. A Study of Piezoelectric Harvesters for Low-Level Vibrations in Wireless Sensor Networks. International Journal of Precision Engineering and Manufacturing. 2013, 14(7) : 1257-1262.

[19] Elesiniotis A., Samson D., Becker T., et al. Investigation of the Performance of Thermoelectric Energy Harvesters under Real Flight Conditions. Journal of Electronic Materials. 2013, 42(7) : 2301-2305.

[20] Wang W. S., Cionca V., Wang N. N. et al. Thermoelectric Energy Harvesting for Building Energy Management Wireless Sensor Networks. International Journal of Distributed Sensor Networks. 2013, 232438.

[21] Jalali F., Khodadoustan S., Ejlali A. Cooperative Hybrid ARQ in Solar Powered Wireless Sensor Networks. Microelectronics Reliability. 2012, 52(12) : 3043-3052.

[22] W. B. Heinzelman, A. P. Chandrakasan, and H. Balakrishnan. Energy-Efficient Communication Protocol for Wireless Microsensor Networks. Proc. of the 33rd Hawaii Int'l Conf. on System Sciences, 2000 : 3005-3014 